\title{
Involvement of epimorphin in the repair of experimental renal fibrosis in mice
}

\author{
Muneharu Yamada ${ }^{1}$, Takashi Oda ${ }^{1}$, Keishi Higashi ${ }^{1}$, Taketoshi Kushiyama ${ }^{1}$, Kazuo Yamakami ${ }^{2}$, Yutaka Sakurai ${ }^{2}$, \\ Yohei Hirai ${ }^{3}$, Kojiro Yamamoto ${ }^{1}$, Toshitake Hyodo ${ }^{1}$, Shigenobu Suzuki ${ }^{1}$, Soichiro Miura ${ }^{1}$ and Hiroo Kumagai ${ }^{1}$
}

Interaction between epithelial cells and mesenchymal cells is essential in normal organ morphogenesis and in tissue repair after injury. Epimorphin, a mesenchymal protein that regulates epithelial morphogenesis through epithelialmesenchymal interactions, has recently attracted attention as an important modulator of tissue repair. In this study we analyzed the role of epimorphin in renal fibrosis. We first found a progressive increase in epimorphin expression corresponding to the progression of renal fibrosis in mice with unilateral ureteral obstruction (UUO). To determine whether this expression has a role in the repair or progression of renal fibrosis, we analyzed a model of renal fibrosis repair, the UUO-release (UUO-R) model. Epimorphin expression was increased at 3 and 7 days after the UUO-R rather than on the day of release, but was decreased at 21 days after the release. Inhibition of endogenous epimorphin with antiepimorphin antibody (MC-1) significantly delayed the repair of fibrosis. When compared with normal-lgG-injected mice, MC-1-injected mice showed significantly decreased renal matrix metalloproteinase (MMP)-2 and MMP-9 expressions by western blotting and increased expression of TGF- $\beta$ and collagen-I mRNA by real-time RT-PCR. Recombinant epimorphin induced prominent increases in MMP-2 and MMP-9 activities in the culture media of renal interstitial fibroblasts in vitro. These findings indicate that epimorphin has a pivotal role in the repair of renal fibrosis by modulating both extracellular matrix (ECM) degradation and its production.

Laboratory Investigation (2010) 90, 867-880; doi:10.1038/labinvest.2010.50; published online 1 March 2010

KEYWORDS: epimorphin; renal fibrosis; repair mechanism; UUO; matrix metalloproteinases

The number of end-stage renal disease (ESRD) patients undergoing dialysis or renal transplant is increasing worldwide and will exceed 2 million by the year $2010,{ }^{1}$ imposing enormous medical and socioeconomic burdens. Renal interstitial fibrosis has an important role in the progression of ESRD. ${ }^{2,3}$ Controlling it is a reasonable therapeutic strategy for preventing or arresting progressive renal disease. The degree of interstitial fibrosis is determined by the balance between the progression and regression of fibrosis. Although mechanisms underlying the progression of renal fibrosis have been studied extensively, mechanisms underlying the repair process have not received as much attention.

An interaction between epithelial cells and mesenchymal cells is essential for normal morphogenesis during organ development and tissue repair after injury. Mesenchymal cells have a key role in these processes by producing or secreting soluble factors, extracellular matrix (ECM) proteins and cell-surface-associated molecules. Epimorphin, also known as syntaxin-2, was originally identified by Hirai et al ${ }^{4}$ in mouse embryonic tissue as a mesenchymal cell-surface-associated protein that has a central role in mesenchymal-epithelial interactions. Previous studies have shown that epimorphin has morphogenetic effects on multiple epithelial tissues, including those of skin, hair follicle, lung, intestine, mammary gland, pancreas, gall bladder and liver. ${ }^{4-10}$ In addition, several investigators have reported changes in the distribution of epimorphin-positive cells in inflammatory diseases of the lung and liver. ${ }^{11-14}$ Epimorphin not only regulates morphogenesis but also functions as a mesenchymal regulator of epithelial repair. ${ }^{10,12,14,15}$

The importance of epithelial-mesenchymal transition in the development of renal fibrosis has been extensively analyzed and characterized. ${ }^{2,16-22}$ However, there have been no reports of studies analyzing the involvement of epimorphin,

\footnotetext{
'Division of Nephrology, Department of Internal Medicine, Saitama, Japan; ${ }^{2}$ Department of Preventive Medicine and Public Health, National Defense Medical College, Saitama, Japan and ${ }^{3}$ Department of Morphoregulation, Institute for Frontier Medical Sciences, Kyoto University, Kyoto, Japan

Correspondence: Dr M Yamada, MD, Department of Nephrology, National Defense Medical College, 3-2 Namiki, Tokorozawa, Saitama 359-8513, Japan.

E-mail: grd1913@ndmc.ac.jp

Received 18 March 2009; revised 31 December 2009; accepted 13 January 2010
} 
which is the key molecule for epithelial-mesenchymal interaction, in renal fibrosis. Moreover, we have not found reports of any studies examining epimorphin expression in diseased kidneys.

In this study we analyzed epimorphin expression in both the progressive and repair phases of renal fibrosis. We used unilateral ureteral obstruction (UUO) in mice as a model of progressive renal fibrosis, ${ }^{22-24}$ and used UUO-release $(\mathrm{UUO}-\mathrm{R})^{25}$ as a model of fibrosis repair. We also evaluated the effect of anti-epimorphin antibody on the repair of renal fibrosis in UUO-R kidneys. In this study we provide data showing that epimorphin contributes to the repair of renal fibrosis by modulating both ECM degradation and ECM production. Targeting epimorphin may have potential as a therapeutic strategy for treating progressive renal disease.

\section{MATERIALS AND METHODS Animals}

Male C57BL/6 mice (6 weeks old) that weighed 20-25 g were purchased from CLEA Japan (Tokyo, Japan). They were given water and standard chow ad libitum. The guidelines of the National Defense Medical College for the care and use of laboratory animals were followed, and the study protocols were approved by the animal ethics committee of the National Defense Medical College.

\section{UUO Model and UUO-R Model}

UUO was performed by double ligation of the left ureter with 3-0 silk under general anesthesia induced by intraperitoneal injection of pentobarbital sodium $(50 \mathrm{mg} / \mathrm{kg} \cdot$ body weight $)$ as described previously. ${ }^{23}$ In sham-operated mice the left ureter was exposed and manipulated but not ligated. Kidneys were harvested for analysis when the mice were killed by exsanguination: UUO mice were killed at 3 or 10 days after surgery ( 5 mice each), and sham-operated mice $(n=5)$ were killed at 10 days after surgery. Both the left kidney (UUO kidney) and right kidney (contralateral kidney) were removed from the UUO mice, but only the left kidney was removed from the sham-operated mice.

UUO-R was achieved as described by Cochrane et al. ${ }^{25} \mathrm{In}$ brief, UUO was performed on the left kidney with the use of a vascular clamp (vessel size, 0.4-1.0 mm; S\&T Fine Science Tools, Foster City, CA, USA), and the clamp was removed under general anesthesia at 10 days after the UUO surgery. UUO-R mice were killed on the day of release or at $3,7,14$ or 21 days after the release (5 mice each). The physiological data of UUO and UUO-R mice are listed in Supplementary Table 1.

\section{Treatment of UUO-R Mice with Anti-Epimorphin Antibody}

At 3 days after UUO-R, the success of the release was checked through an abdominal incision. The UUO-R mice in which release was successful were randomly assigned to one of two groups. One group of five received every-other-day intraperitoneal injections ( $15 \mu \mathrm{g} / \mathrm{g}$ body weight) of rat monoclonal anti-epimorphin antibody (MC-1) prepared as described previously by Hirai et al. ${ }^{4}$ The control group of five received intraperitoneal injections of the same dose of normal rat IgG (Sigma-Aldrich, St Louis, MO, USA). All mice were killed at 14 days after UUO-R, and the left kidney of each mouse was harvested.

In all experiments, the harvested kidneys were immediately decapsulated and cut into several pieces that were either fixed in $10 \%$ neutral buffered formalin and embedded in paraffin for histological analysis or stored at $-80^{\circ} \mathrm{C}$ for later analysis.

\section{Renal Histology, Immunoperoxidase Staining and Immunofluorescence (IF) Staining}

Sections (4- $\mu \mathrm{m}$ thick) of formalin-fixed paraffin-embedded tissue from the kidneys of sham-operated, UUO and UUO-R mice were subjected to Masson's trichrome staining. Interstitial macrophages, myofibroblasts and proliferating cells in paraffin-embedded sections of kidneys of UUO-R mice treated with MC-1 were evaluated by immunoperoxidase staining for macrophages, $\alpha$-SMA and proliferating cell nuclear antigen (PCNA) as described previously. ${ }^{23,26}$

Cryostat sections (5- $\mu \mathrm{m}$ thick) of fresh frozen kidney were used for IF staining. Kidney sections from sham-operated, UUO and UUO-R mice were used for epimorphin staining, and kidney sections from MC-1-treated UUO-R mice were used for E-cadherin staining. After fixation with $4 \%$ paraformaldehyde and blocking with blocking buffer (StartingBlock, PIERCE, Rockford, IL, USA), sections were incubated with MC-1 or anti-E-cadherin antibody overnight at $4{ }^{\circ} \mathrm{C}$ before being incubated with a secondary antibody for $1 \mathrm{~h}$ at room temperature. For the epimorphin staining, the MC-1-positive interstitial area in each of three-to-six random non-overlapping cortical fields at $\times 200$ magnification was evaluated using a digital image analysis system (Lumina Vision, version 2.04; Mitani, Fukui, Japan) and expressed as a percentage of the total area excluding glomeruli and large vessels. For the E-cadherin staining, the proportion of E-cadherin-positive tubules among all the tubules in five random non-overlapping cortical fields at $\times 200$ magnification was calculated.

In some frozen sections of kidney tissues obtained 10 days after UUO, three combinations of multiple stainings for epimorphin and other cell markers (laminin, vimentin, $\alpha$-SMA, matrix metalloproteinase (MMP)-2 and MMP-9) were performed to identify the epimorphin-positive interstitial area. One combination was triple staining for epimorphin, vimentin and laminin; the second was the double staining for epimorphin and $\alpha$-SMA and the third was double staining for epimorphin and either MMP-2 or MMP-9. The sections were incubated with primary antibodies overnight at $4{ }^{\circ} \mathrm{C}$ and with secondary antibodies for $1 \mathrm{~h}$ at room temperature. For double stainings we counterstained the nuclei with Hoechst 33342 (Molecular Probes, Eugene, OR, USA). Details of the primary and secondary 
Table 1 Primary antibodies used in this study

\begin{tabular}{llll}
\hline Antibody type & Clone & Conjugate & Supplier \\
\hline Rat anti-macrophage & F4/80 & - & Serotec, Oxford, UK \\
Mouse anti- $\alpha$-SMA & 1 A4 & EPOS & DAKO, Carpinteria, CA, USA \\
Mouse anti- $\alpha$-SMA & 1 A4 & FITC & Sigma-Aldrich, St Louis, MO, USA \\
Mouse anti-PCNA & PC-10 & EPOS & DAKO \\
Goat anti-E-cadherin & - & - & R\&D System, Minneapolis, MN, USA \\
Guinea-pig anti-vimentin & - & - & PROGEN Biotechnik GmbH, Heidelberg, Germany \\
Rabbit anti-laminin & - & - & Sigma-Aldrich \\
Rabbit anti-MMP-2 & - & HRP & Biomol International LP, Plymouth Meeting, PA, USA \\
Rabbit anti-MMP-9 & -19 & Biomol International LP \\
Rabbit anti- $\beta$-actin & & Santa Cruz Biotechnology, Santa Cruz, CA, USA
\end{tabular}

$\alpha$-SMA, $\alpha$-smooth muscle actin; EPOS, enhanced polymer one-step staining; FITC, fluorescein isothiocyanate; PCNA, proliferating cell nuclear antigen; MMP, matrix metalloproteinase; HRP, horseradish peroxidase.

Table 2 Combinations of primary and secondary antibodies used for IF staining

\begin{tabular}{llll}
\hline Target antigen for primary antibody & & Secondary antibody \\
\cline { 2 - 4 } & Antibody type & Label & Supplier \\
\hline E-cadherin & Chicken anti-goat lgG & AF 488 & Molecular Probes, Eugene, OR, USA \\
Epimorphin & Goat anti-rat lgG & AF 488 & Molecular Probes \\
& Donkey anti-rat lgG & AF 594 & Molecular Probes \\
Vimentin & Goat anti-guinea pig lgG & AF 594 & Molecular Probes \\
Laminin & Goat anti-rabbit lgG & AF 350 & Molecular Probes \\
$\alpha$-SMA & Goat anti- FITC & FITC & GeneTex, San Antonio, TX, USA \\
MMP-2 & Goat anti-rabbit lgG & AF 594 & Molecular Probes \\
MMP-9 & Goat anti-rabbit lgG & AF 594 & Molecular Probes
\end{tabular}

AF, Alexa Fluor.

antibodies are listed in Tables 1 and 2. Digital images of stained sections were obtained with a confocal microscope (Zeiss LSM 510; Carl Zeiss, Göttingen, Germany).

\section{Western Blot Analysis}

Whole kidney homogenates were prepared, and western blot analysis was performed as described previously. ${ }^{27}$ Protein concentrations were measured using the Bio-Rad protein assay. Protein samples $(30 \mu \mathrm{g})$ were separated by SDS-PAGE on $10 \%$ acrylamide gels and transferred onto nitrocellulose membranes. The primary antibodies used for western blotting were MC-1 antibody, anti-MMP-2 and anti-MMP-9 antibody, and EPOS reagents of 1A4 and PC-10 (see details in Table 1). Bands were detected using enhanced chemiluminescence kit (ECL Plus, GE Healthcare UK, Buckinghamshire, UK). Equal loading and transfer of samples were confirmed and adjusted according to the $\beta$-actin signal. The density of each band was quantified with CS Analyzer, version 2.0 (Atto, Tokyo, Japan).

\section{Real-time RT-PCR}

Total RNA was extracted from the kidney tissues using the RNeasy Mini kit (Qiagen, Hilden, Germany). RNA was quantified by absorbance at $260 \mathrm{~nm}$. A $1-\mu \mathrm{g}$ aliquot of total RNA was reverse-transcribed into first-strand cDNA with random primers using the PrimeScript RT Reagent Kit (total volume, $20 \mu \mathrm{l}$; Takara, Shiga, Japan). The constructed cDNA served as a template for real-time PCR. We used pre-designed TaqMan Gene Expression Assays for mouse epimorphin (syntaxin-2), collagen I, collagen III, TGF- $\beta 1$ and GAPDH (Applied Biosystems, Foster City, CA, USA). PCR was performed in triplicate using the ABI Prism 7900 sequence 
detection system (Applied Biosystems) in 96-well microtiter plates in a final volume of $20,10 \mu \mathrm{l}$ of which was TaqMan Universal PCR Master Mix (Applied Biosystems), $1 \mu \mathrm{l}$ of which was pre-designed primer/probe mix and $9 \mu$ l of which was cDNA. Reaction conditions consisted of pre-incubation at $50{ }^{\circ} \mathrm{C}$ for $2 \mathrm{~min}, 95^{\circ} \mathrm{C}$ for $10 \mathrm{~min}$ and then 40 cycles at $95^{\circ} \mathrm{C}$ for $15 \mathrm{~s}$ and $60^{\circ} \mathrm{C}$ for $1 \mathrm{~min}$. Relative expression levels were calculated by using the $\Delta \Delta \mathrm{Ct}$ method.

\section{Evaluation of Renal Fibrosis}

The degree of interstitial fibrosis in the UUO-R kidney was evaluated using image analysis software (Lumina Vision; Mitani) to measure the light-blue-stained areas after Masson's trichrome staining as reported previously. ${ }^{28,29}$ In the experiment in which UUO-R mice were given anti-epimorphin antibody, degrees of renal fibrosis were evaluated histologically by picrosirius red staining and biochemically by total collagen assay as described previously. ${ }^{27}$ Picrosirius red staining was performed on paraffin-embedded sections and the stained sections were observed by polarized-light microscopy. For the total collagen assay, the hydroxyproline concentration in hydrolysates of precisely weighed frozen kidney samples was measured chemically. Total collagen was assumed to contain $12.7 \%$ hydroxyproline by weight. Results are expressed as micrograms of collagen per milligram kidney weight.

\section{Gel Zymography for Plasminogen Activator (PA) and Plasmin Activity}

Intrarenal PA and plasmin activities were analyzed by plasminogen-casein zymography and casein gel zymography, respectively, as described previously. ${ }^{27,30}$ In brief, kidney protein samples $(10 \mu \mathrm{g}$ protein for PA and $40 \mu \mathrm{g}$ protein for plasmin) isolated for western blotting were separated under nonreducing conditions on $10 \%$ SDS polyacrylamide gels containing casein $(2 \mathrm{mg} / \mathrm{ml})$ either with (for PA) or without (for plasmin) $10 \mu \mathrm{g} / \mathrm{ml}$ of plasminogen (Sigma-Aldrich). After a wash in $2.5 \%$ Triton X-100, gels were incubated with substrate buffer $(0.1 \mathrm{M}$ glycine, $\mathrm{pH} 8.3)$ at $37^{\circ} \mathrm{C}$. Incubation time was $16 \mathrm{~h}$ for the plasminogen-casein gel and $36 \mathrm{~h}$ for the casein gel. After staining with Coomassie blue, the density of each lytic band was quantified with CS Analyzer, version 2.0 (Atto).

\section{Cell Culture, Treatments and Gelatin Gel Zymography}

NRK-49F (interstitial fibroblast cell line of normal rat kidney) and NRK-52E (normal rat kidney tubular epithelial cell line) cells were purchased from the American Type Culture Collection. Cells were cultured in Dulbecco's modified Eagle's medium supplemented with $1 \%$ nonessential amino acids and $5 \%$ fetal bovine serum. Before treatment, cells were seeded onto 24-well plates and maintained in serum-free medium (Cosmedium-001, Cosmobio, Tokyo, Japan) until $70 \%$ confluence was reached. Recombinant epimorphin (final concentration of $20 \mu \mathrm{g} / \mathrm{ml}$ ) or vehicle was added to the culture medium, and the supernatants were collected at various time points $(8,16$ and $24 \mathrm{~h})$ after treatment. The recombinant epimorphin was prepared as described previously by Hirai et al. ${ }^{31}$ The collected mediums were concentrated 15-fold with centrifugal filter units (UltrafreeMC; Millipore, Bedford, MA, USA). MMP activities in each concentrated sample were analyzed by gel zymography similar to that described above except that $1 \mathrm{mg} / \mathrm{ml}$ gelatin instead of casein was added to the gel and the substrate buffer was $50 \mathrm{mM}$ Tris and $20 \mathrm{mM} \mathrm{CaCl}_{2}$ (pH 7.6). The density of each lytic band was quantified with CS Analyzer version 2.0 (Atto). Total RNA was extracted from NRK-49F cells that were either treated or not treated by recombinant epimorphin, and the MMP-9 mRNA levels were evaluated by real-time RT-PCR with the pre-designed primer/probe set of rat MMP-9 (Applied Biosystems) as described above.

\section{Statistical Analysis}

Data are shown as means \pm s.e. Differences between groups were subjected to one-way or two-way analysis of variance (ANOVA). Fisher's protected least significant difference test was used for post hoc analysis and $P<0.05$ was considered statistically significant. All statistical analyses were performed using StatView, version 5.0 (SAS Institute, Cary, NC, USA).

\section{RESULTS \\ Epimorphin Expression in Kidneys of Sham-Operated and UUO Mice}

The UUO kidneys of UUO mice showed progressive tubulointerstitial mononuclear cell infiltration and interstitial fibrosis, whereas the contralateral kidneys of UUO mice and the kidneys of sham-operated mice showed minimal cell infiltration and no interstitial fibrosis (Figure 1a, upper panels). IF staining for epimorphin was positive in the glomerular mesangium, vessel walls and interstitial area in the kidneys of sham-operated mice, and IF staining for epimorphin in the contralateral kidneys of UUO mice was essentially the same as that in the kidneys of sham-operated mice. Tubulointerstitial epimorphin staining in the UUO kidneys of UUO mice was significantly stronger than that in the UUO kidneys of sham-operated mice, but the intensity of glomerular epimorphin staining did not differ between them. Tubulointerstitial epimorphin staining was stronger and more widespread in UUO kidneys harvested 10 days after UUO than in UUO kidneys harvested 3 days after UUO (Figure 1a, lower panels). Such an increase in epimorphin expression was confirmed by western blotting (protein levels) (Figure 1b) and by real-time RT-PCR (mRNA levels; Figure 1c).

Tubulointerstitial epimorphin expression in UUO kidneys at 10 days after UUO was characterized using confocal microscopy with triple staining for laminin, epimorphin and vimentin and for nuclei, $\alpha$-SMA and epimorphin (Figure 2). Vimentin was used as a mesenchymal marker and $\alpha$-SMA was used as a marker for myofibroblasts. Laminin was used mainly as a marker of tubular basement membrane (TBM) 
a
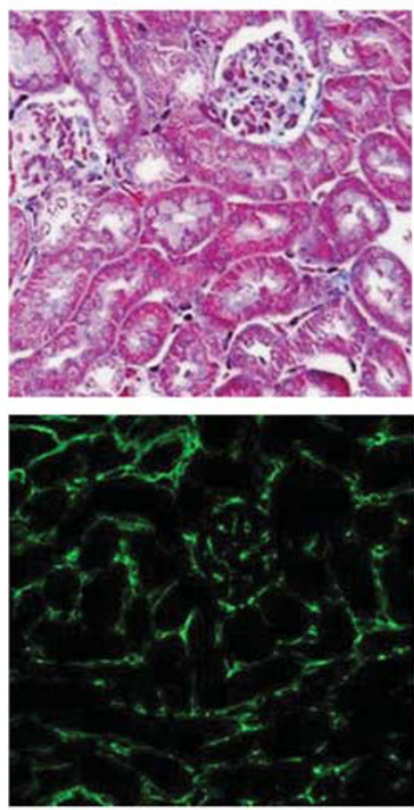

b

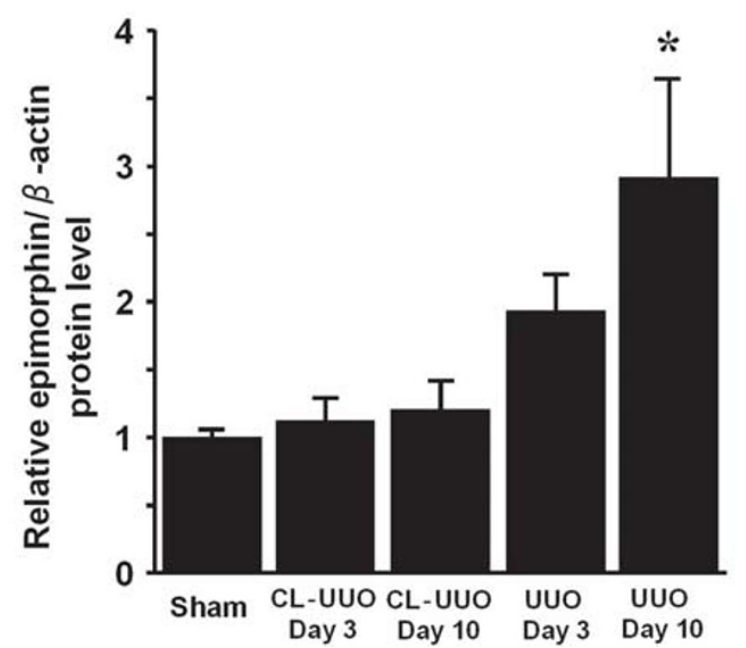

UUO Day 3
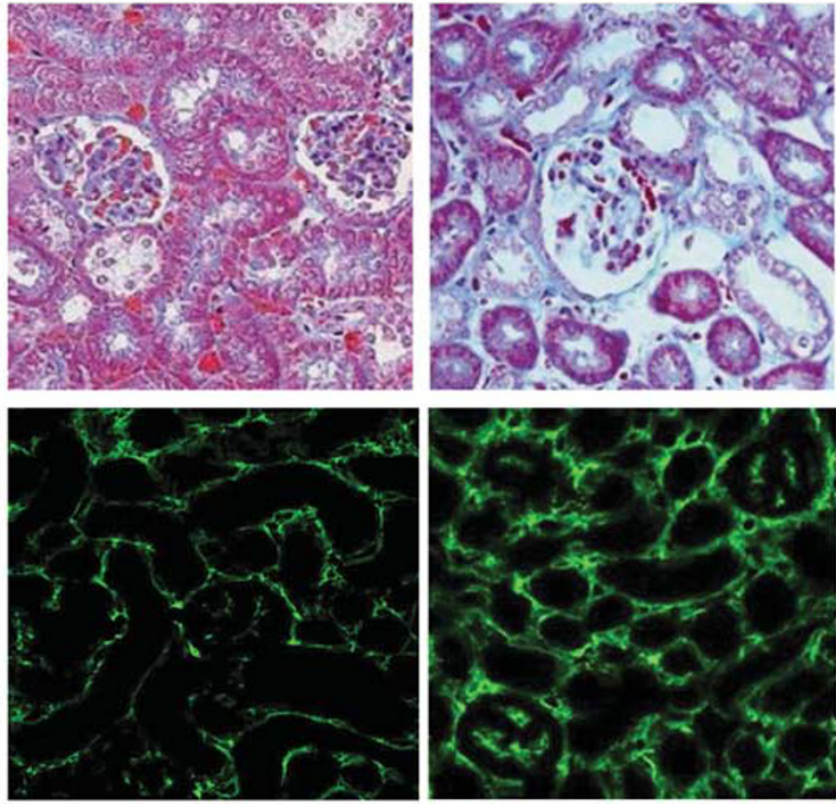

UUO Day 10
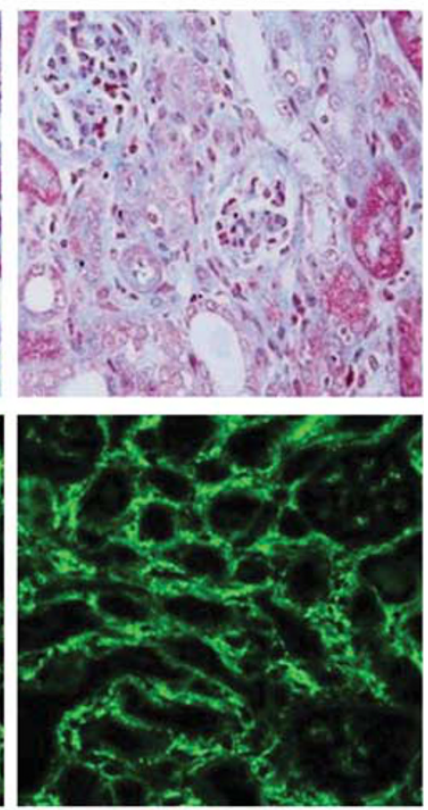

C

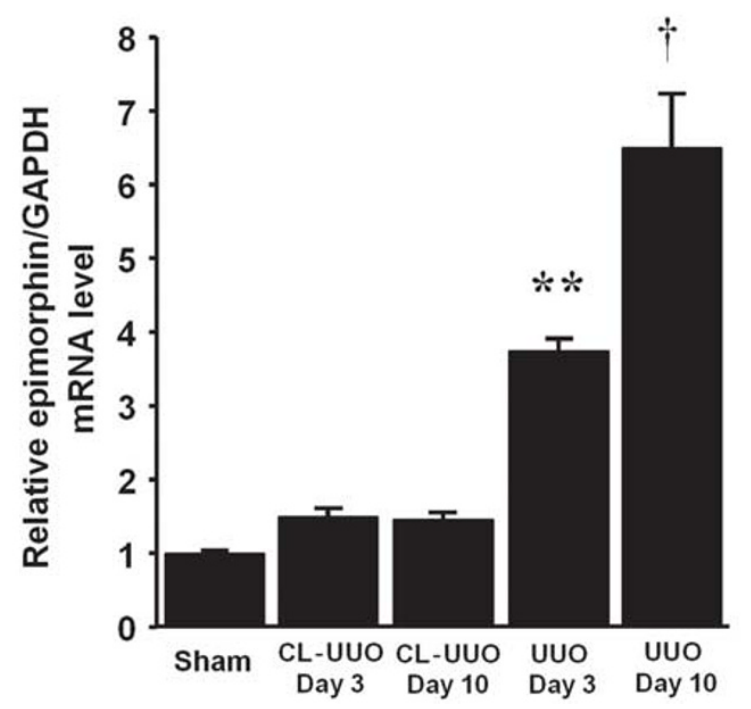

Figure 1 Epimorphin expression in kidneys of sham-operated and unilateral ureteral obstruction (UUO) mice. (a) Representative photomicrographs showing progressive renal interstitial fibrosis and epimorphin expression in mouse kidney subjected to UUO. (Upper panels) Masson's trichrome staining of renal tissues from the kidney of a sham-operated mouse, the contralateral (CL) kidney of a UUO mouse on day 10, and from UUO kidneys on days 3 and 10. (Lower panels) Corresponding immunofluorescence staining for epimorphin. Original magnification $\times 400$. (b) Representative western blots for epimorphin in kidneys of sham-operated mice and in $\mathrm{CL}$ and UUO kidneys at 3 and 10 days after UUO. Lower graph shows corresponding epimorphin protein levels relative to $\beta$-actin levels (mean \pm s.e., $n=5,{ }^{*} P<0.01$ vs sham and vs $\mathrm{CL}$ kidneys on days 3 and 10). (c) Epimorphin mRNA levels assessed by real-time RT-PCR (mean \pm s.e., $n=5,{ }^{* *} P<0.001$ vs sham and vs CL kidneys on days 3 and $10,{ }^{\dagger} P<0.0001$ vs sham and vs CL kidneys on days 3 and 10 and UUO kidney on day 3 ).

but was also found on glomerular mesangium and glomerular basement membrane. Merged images revealed that tubulo-interstitial epimorphin staining was not on tubular epithelial cells but in the interstitial area just around the TBM and was intermingled with vimentin-positive or $\alpha$-SMApositive cells. The epimorphin-positive portions of the interstitium colocalized well with $\alpha$-SMA-positive cells (Figure 2b) but did not colocalize with vimentin-positive 
a
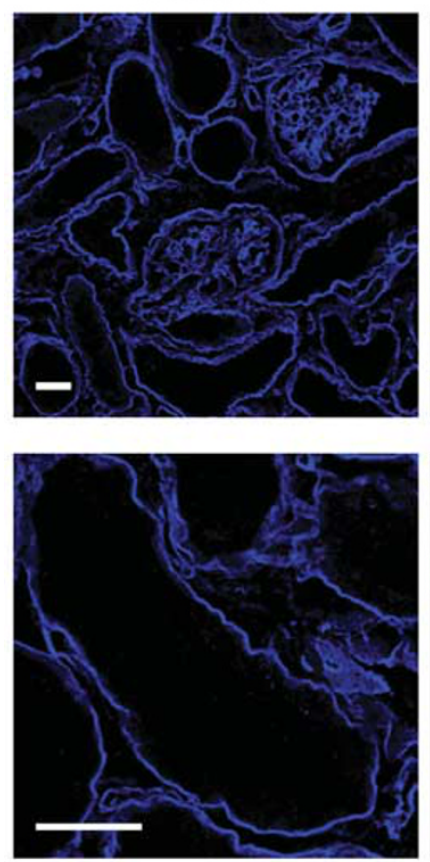

b

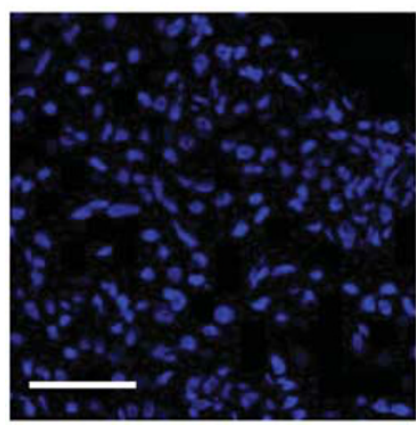

Epimorphin
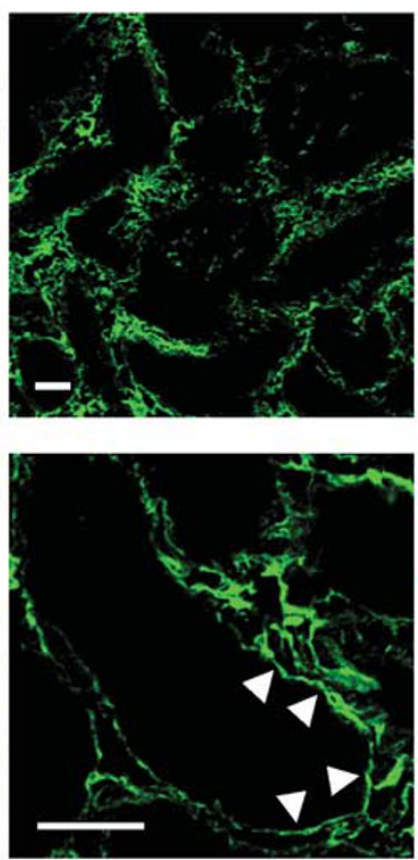

$\alpha$ - SMA

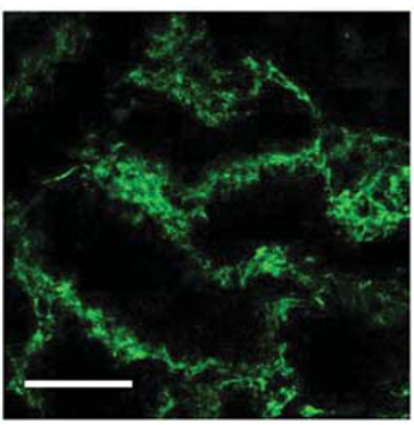

Vimentin
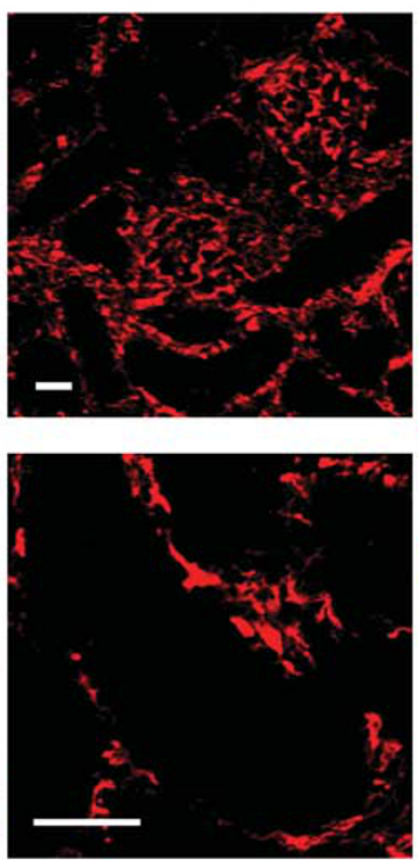

Epimorphin

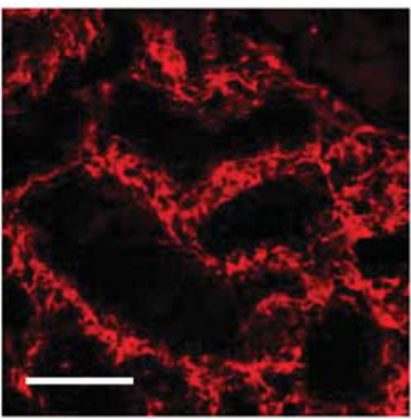

Merged image
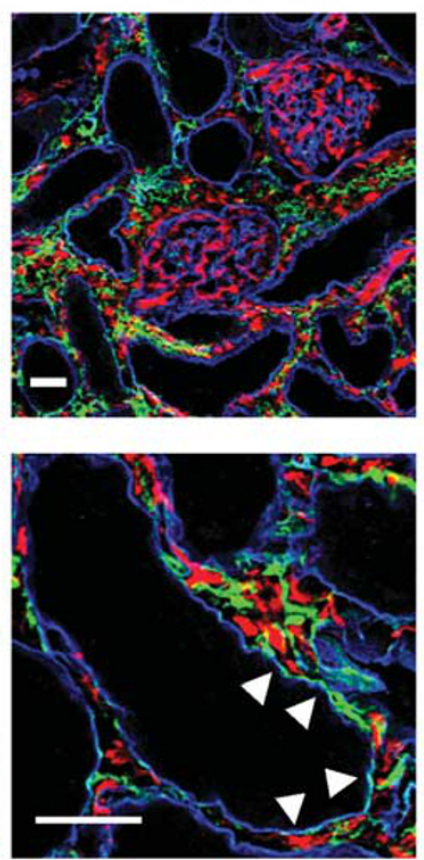

Merged image

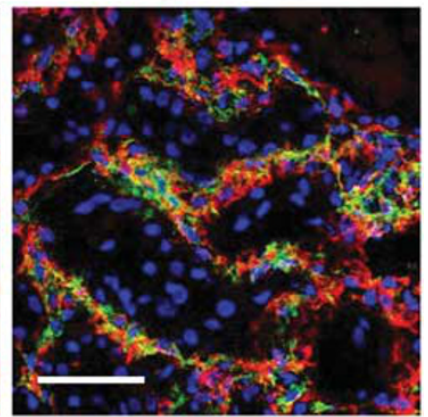

Figure 2 Confocal microscopy images of multiple immunofluorescence staining in mouse kidney at 10 days after UUO (scale bar $=20 \mu \mathrm{m}$.).

(a) Representative low-power (upper panels) and high-power (lower panels) photomicrographs of triple staining for laminin, epimorphin and vimentin. Note the epimorphin-positive interstitial area around tubular basement membrane (arrows) and that interstitial epimorphin-positive and vimentin-positive cells are in close proximity but not colocalized. (b) Representative photomicrographs of multiple staining for nuclei, $\alpha$-smooth muscle actin ( $\alpha$-SMA) and epimorphin. Merged image revealed high colocalization of epimorphin-positive portion and $\alpha$-SMA-positive cells in the interstitium of UUO kidney.

cells or with cells positive for another fibroblast marker, the fibroblast-specific protein-1 (FSP-1; data not shown). Thus, tubulo-interstitial epimorphin was localized mainly on interstitial myofibroblasts and in the interstitial ECM, especially around the TBM. On the other hand, glomerular epimorphin colocalized with laminin in mesangial area of glomeruli (Supplementary Figure 1).

\section{Epimorphin Expression in the UUO-R Model}

We followed the method of Cochrane et al ${ }^{25}$ (mice of the same strain, similar body weight, use of the same clamps and same period of ureter obstruction) for the UUO-R model, but we found persistent hydronephrosis in some mice even after removal of the ureter clamps, probably due to the organization of obstructed tissues. Changing the clamped portion of the ureter (upper, middle or lower portion), the direction of the clamp (toward the center or toward the side) and the clamping angle did not reliably prevent this problem from occurring, and hence we checked the release of the ureteral obstruction by opening the abdomen of all mice at 3 days after removal of the clamps and used only those mice in which the release was successful.

Masson's trichrome staining revealed progressive regression of the interstitial fibrosis over time in UUO-R kidneys (Figure 3a, upper panels, and $3 \mathrm{~b}$ ). IF staining for epimorphin was significantly stronger at 3 and 7 days after the release than it was on the day of release (day 0 ) and was significantly reduced on day 21 (Figure 3a, lower panels, and 3c). 
a

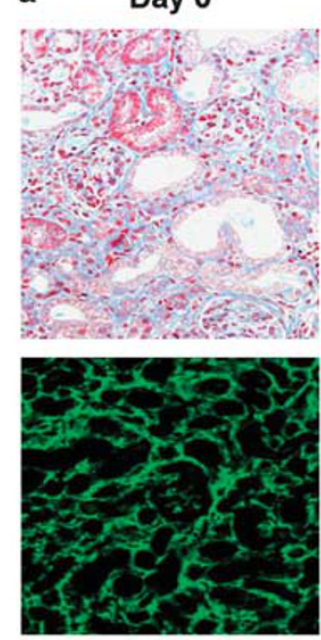

Day 3
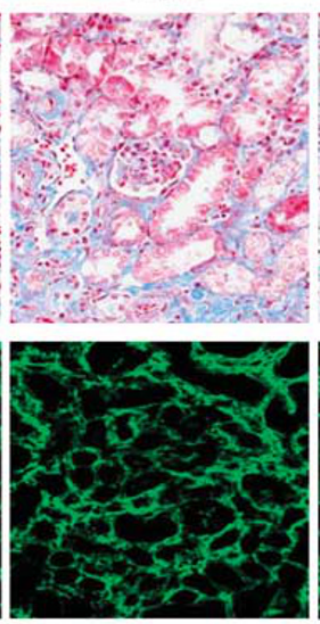

Day 7
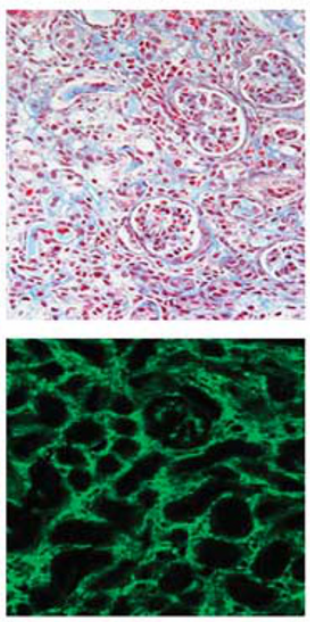

Day 14
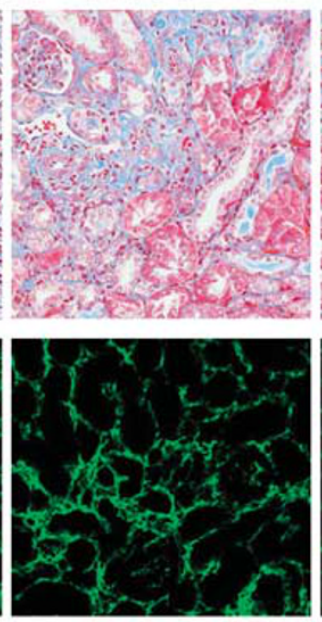

Day 21
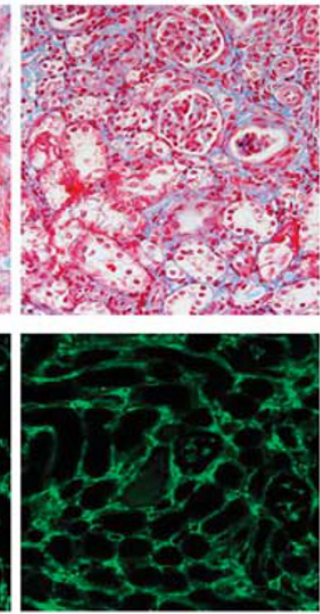
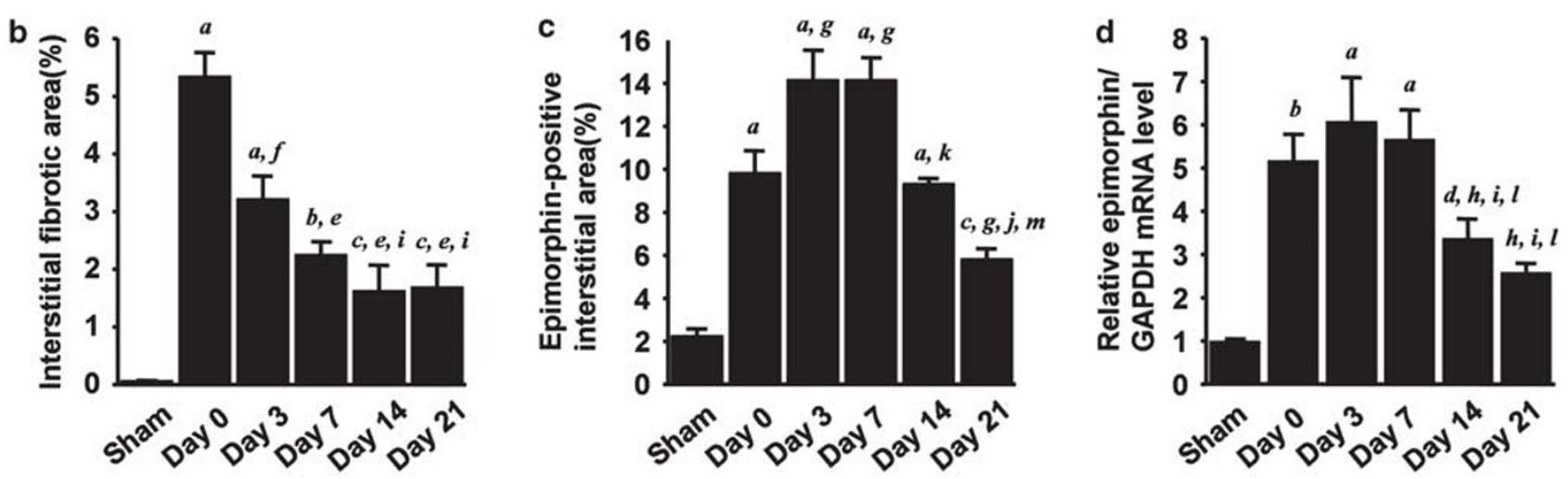

Figure 3 Time sequence of interstitial fibrosis and epimorphin expression in mouse kidney after UUO release (UUO-R). (a) Representative photomicrographs of Masson's trichrome staining of kidney tissue on the day of UUO-R (day 0) and 3 (day 3), 7 (day 7), 14 (day 14) and 21 (day 21) days later. Lower panels show corresponding immunofluorescence staining for epimorphin. Original magnification $\times 400$. (b) Graph showing relative percentages of light-bluestained interstitial areas in kidney cortices after Masson's trichrome staining. (c) Graph showing relative percentages of epimorphin-positive interstitial areas in kidney cortices. (d) Graph showing relative epimorphin mRNA expression (normalized to GAPDH mRNA expression) as assessed by real-time RT-PCR. Values in bar graphs are means \pm s.e., $n=5$ per group. (a) $P<0.0001$ vs sham, (b) $P<0.001$ vs sham, (c) $P<0.01$ vs sham, (d) $P<0.05$ vs sham, (e) $P<0.0001$ vs day 0 , (f) $P<0.001$ vs day 0 , (g) $P<0.01$ vs day 0 , (h) $P<0.05$ vs day 0 , (i) $P<0.01$ vs day 3 , (j) $P<0.0001$ vs days 3 and 7 , (k) $P<0.001$ vs days 3 and 7 , (l) $P<0.01$ vs day 7 and $(\mathrm{m}) P<0.05$ vs day 14 .

Results of real-time RT-PCR for epimorphin mRNA (Figure 3d) were consistent with the histological observations of IF staining.

\section{Repair of Renal Fibrosis was Retarded by Neutralization of Endogenous Epimorphin after UUO-R}

To determine the roles of enhanced epimorphin expression in the early phase after UUO-R, we examined the effect of treating the mice with a monoclonal anti-epimorphin antibody (MC-1). We found a significant delay in recovery from interstitial fibrosis in the MC-1-treated mice compared with that in mice treated with normal rat IgG (control) at 14 days after UUO-R, according to picrosirius red staining and total collagen assay $(12.33 \pm 0.97$ vs $9.49 \pm 0.62 \mu \mathrm{g} / \mathrm{mg}$ kidney weight, $P<0.05$; Figure $4 \mathrm{a}$ and $\mathrm{b}$ ).

The molecular mechanisms underlying the difference in the degree of interstitial fibrosis between MC-1-treated mice and control mice were analyzed in terms of ECM production and ECM degradation. With respect to ECM production, real-time RT-PCR revealed that TGF- $\beta$, collagen $\mathrm{I}$ and collagen III mRNAs were expressed more strongly in MC-1treated mice than in control mice, though the difference in the expression of collagen III mRNA was not statistically significant (Figure 5). With respect to ECM degradation, western blotting for MMP-2 and MMP-9 revealed dramatic decreases in the expression of these proteins (approximately 0.27 and 0.14 times, respectively) in the MC-1-treated mice (Figure $6 \mathrm{a}$ and $\mathrm{b}$ ). The activities of intrarenal plasmin and PAs, in contrast, were significantly higher in the MC-1treated mice (Figure $6 \mathrm{c}$ and $\mathrm{d}$ ).

According to immunohistochemistry and western blotting, the severity of interstitial infiltration of myofibroblasts and macrophages and the degree of cell proliferation were greater in $\mathrm{MC}-1$-treated mice than in control mice (Figure $7 \mathrm{a}-\mathrm{c}$ ). 
a

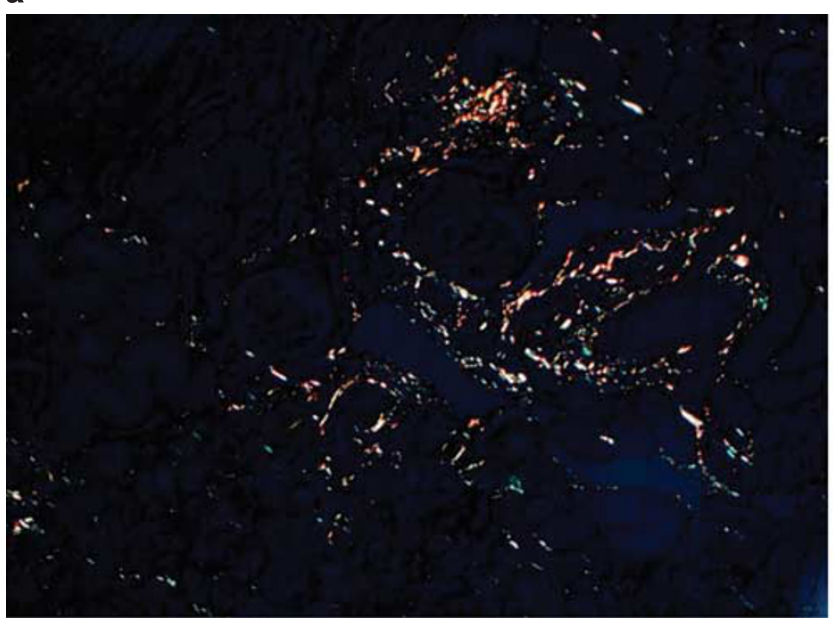

Control

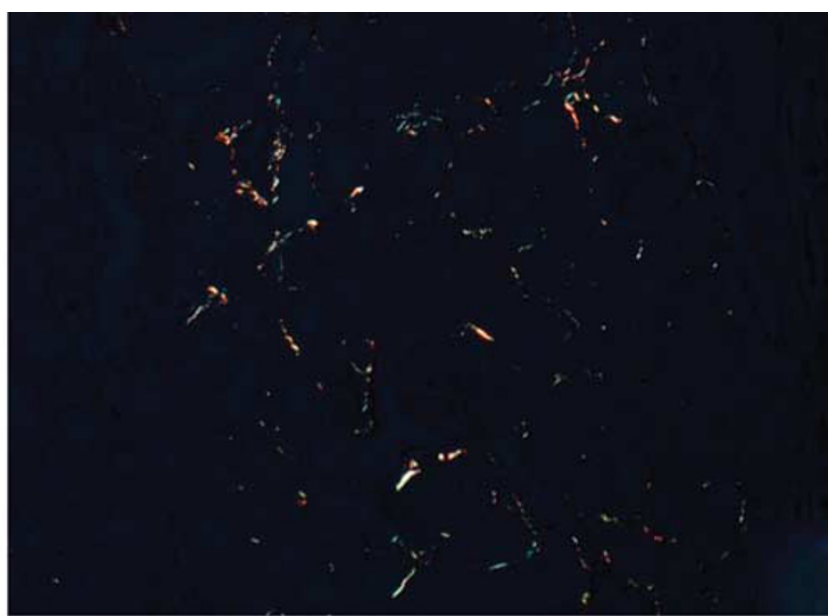

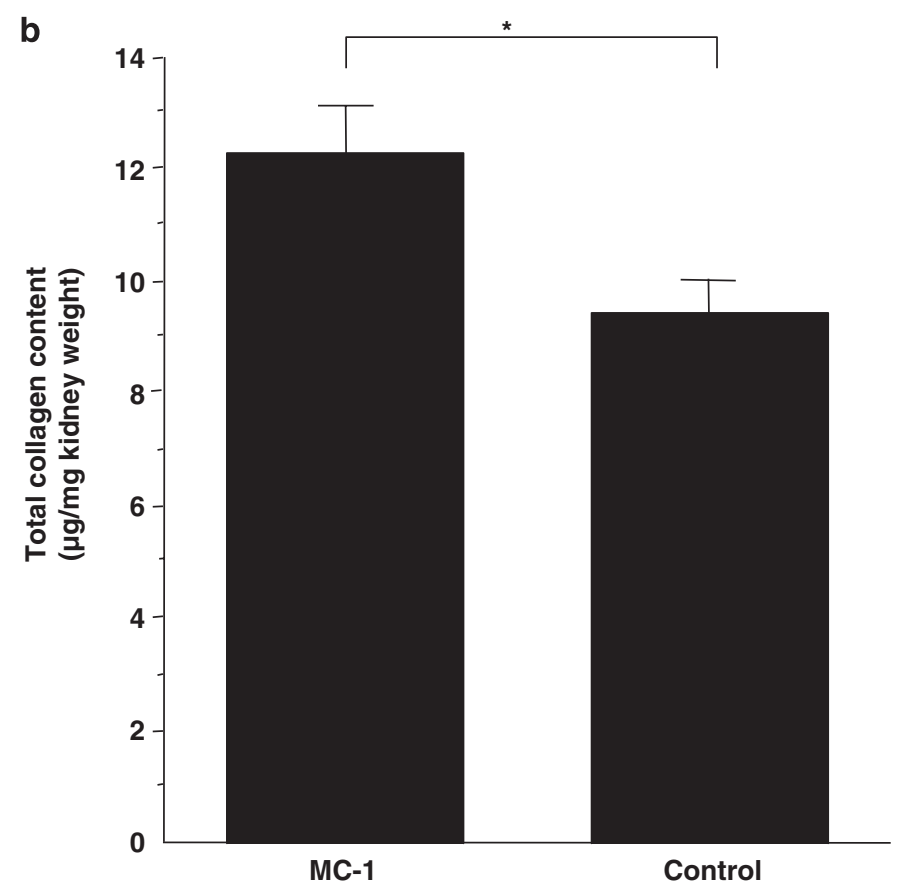

Figure 4 Effect of anti-epimorphin antibody (MC-1) on renal interstitial fibrosis after UUO-R. (a) Photomicrographs of picrosirius red staining of renal tissues from mice treated with MC-1 or normal rat IgG (control) for 14 days after UUO-R. Original magnification $\times 400$. (b) Total collagen content of kidneys from the MC- 1 and control groups (mean \pm s.e., $n=5,{ }^{*} P<0.05$ ).

Although the staining intensity for E-cadherin varied greatly between different segments of the tubular epithelial cells, most cortical tubular epithelial cells were positive for E-cadherin. The percentage of tubules positive for E-cadherin in the MC-1-treated mice was significantly lower than that in the control mice (Figure 8).

\section{Recombinant Epimorphin Induces MMP-2 and MMP-9 Secretions in Renal Interstitial Fibroblasts In Vitro}

Gelatin zymography revealed no MMP-9 activity and only weak MMP-2 activity in the culture media of unstimulated
NRK-52E cells. Adding recombinant epimorphin induced weak MMP-9 activity but did not change MMP-2 activity. Prominent MMP-2 and MMP-9 activities were, however, observed in the culture media of unstimulated NRK-49F cells, and in these cells recombinant epimorphin upregulated MMP-9 activity strongly and upregulated MMP-2 activity weakly (Figure 9). Indeed, for both MMP-2 and MMP-9 activities of NRK-49F cells, a significant effect was found in epimorphin stimulation $(P<0.0001)$ and in time sequences (for MMP-2, $P<0.05$ between 8 and $16 \mathrm{~h}$ and between 8 and $24 \mathrm{~h}$; for MMP-9, $P<0.01$ between 8 and $24 \mathrm{~h}$ ) by two-way 

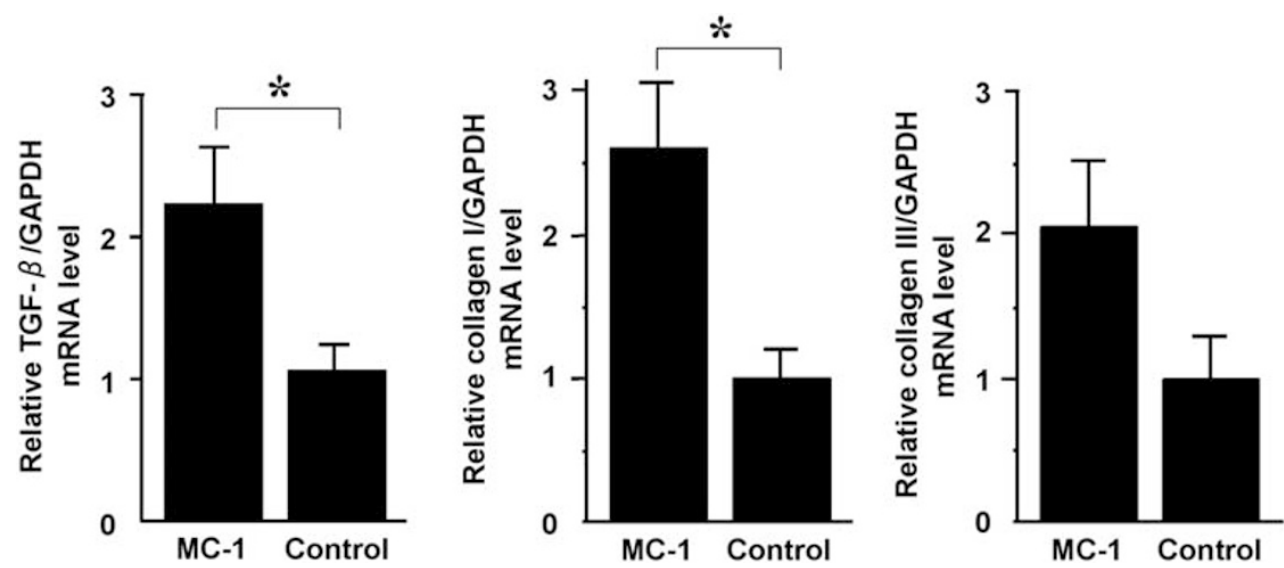

Figure 5 Relative TGF- $\beta$, collagen I and collagen III mRNA expression assessed by real-time RT-PCR in UUO-R kidneys treated with MC-1 or control for 14 days. mRNA expression relative to GAPDH mRNA expression (mean \pm s.e., $n=4,{ }^{*}<<0.05$ ).

a
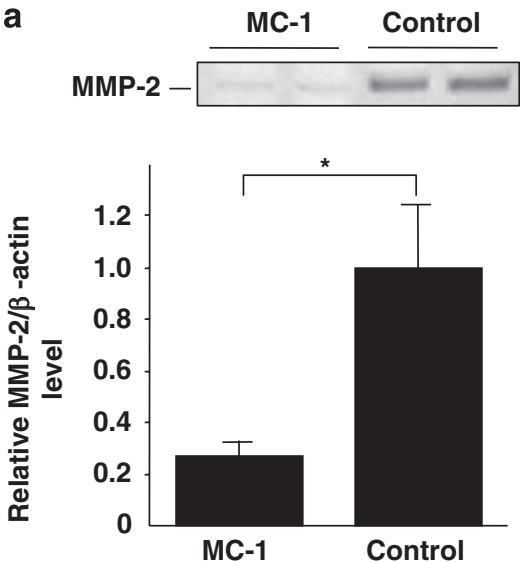

C
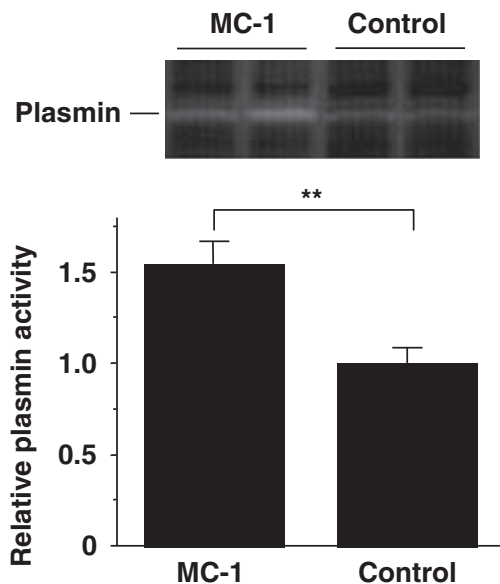

b
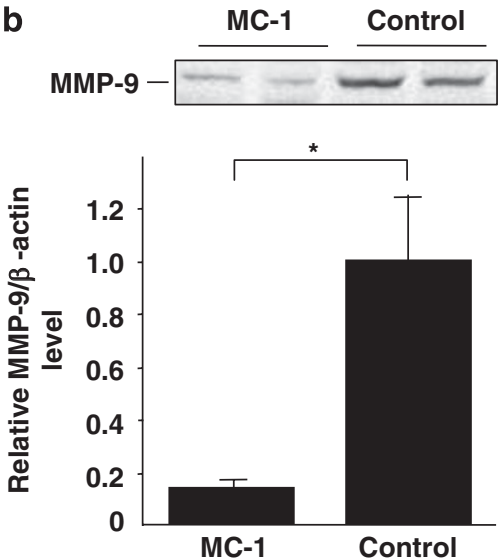

d
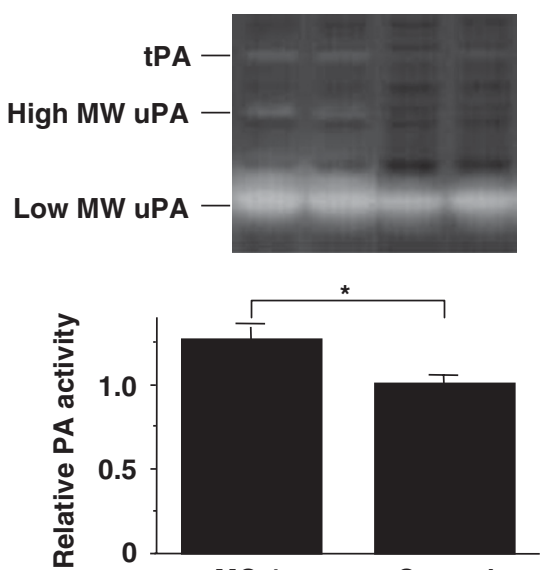

MC-1

Control

Figure 6 Intrarenal protease levels in UUO-R kidneys treated with MC-1 or control for 14 days. (a) Western blot of MMP-2 and graph of relative MMP-2 level relative to $\beta$-actin level (mean \pm s.e., $n=5,{ }^{*}>0.05$ ). (b) Western blot of MMP-9 and graph of MMP-9 level relative to $\beta$-actin level (mean \pm s.e., $n=5$, ${ }^{*} P<0.05$ ). (c) Casein gel zymography and graph of relative plasmin activity assessed from the density of each lytic band (mean \pm s.e., $n=5$, $* * P<0.01$ ). (d) Plasminogen-casein gel zymography and graph of relative total plasminogen activator (PA) activity (sum of tissue-type PA (tPA), high-molecular-weight urokinase-type PA (HMW uPA) and low-molecular-weight UPA (LMW uPA)) assessed from the density of each lytic band (mean \pm s.e., $n=5, * P<0.05$ ). 


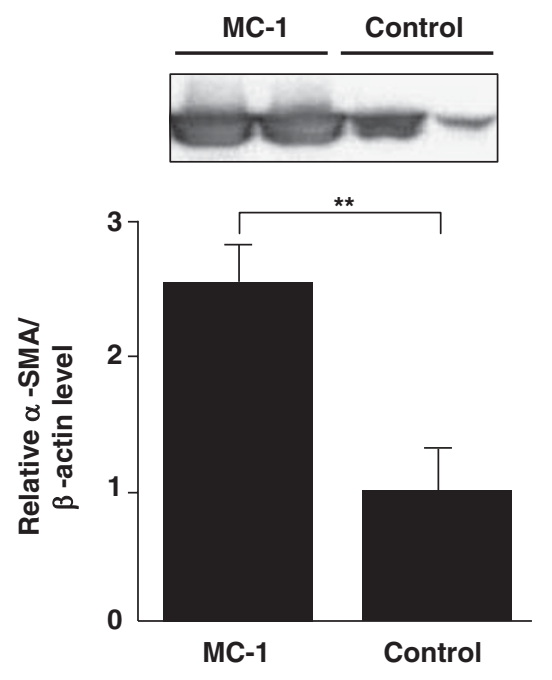

b
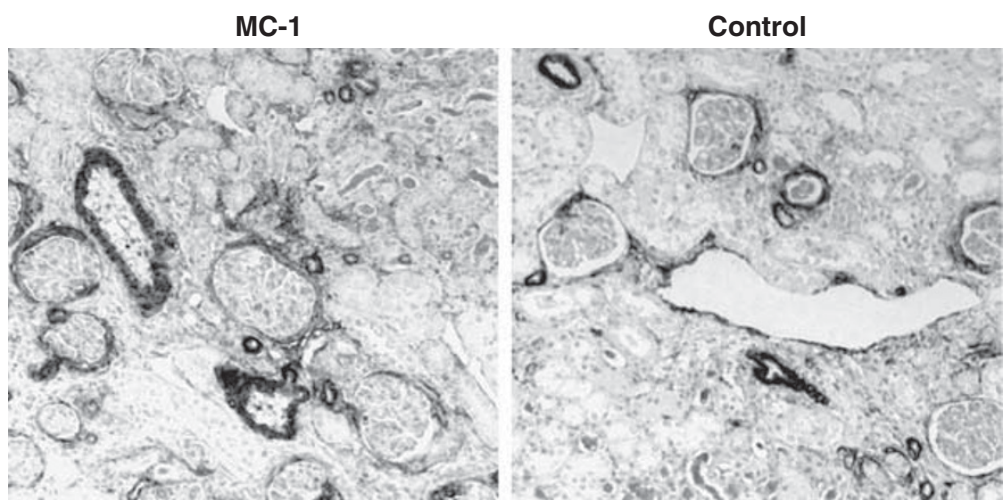

MC-1

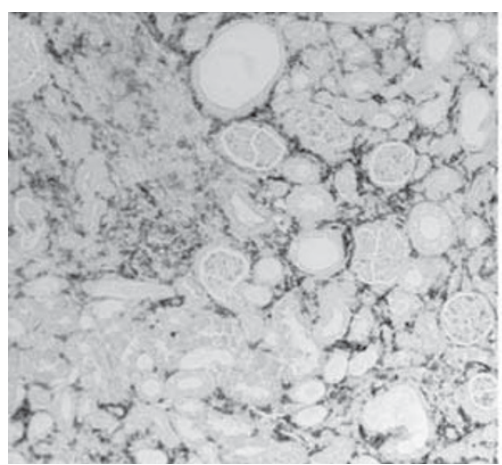

C

MC-1

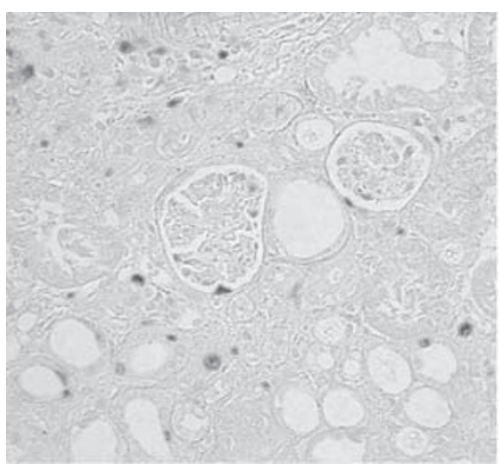

Control

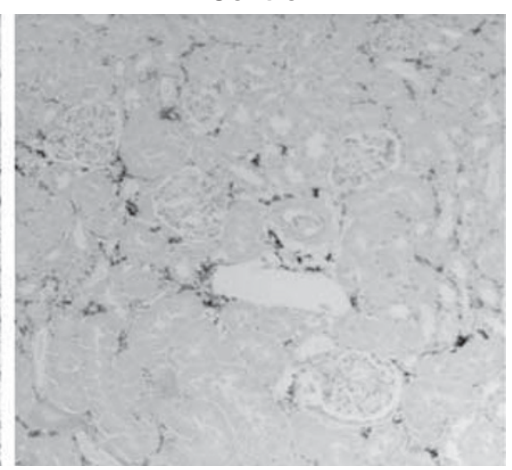

\section{.}

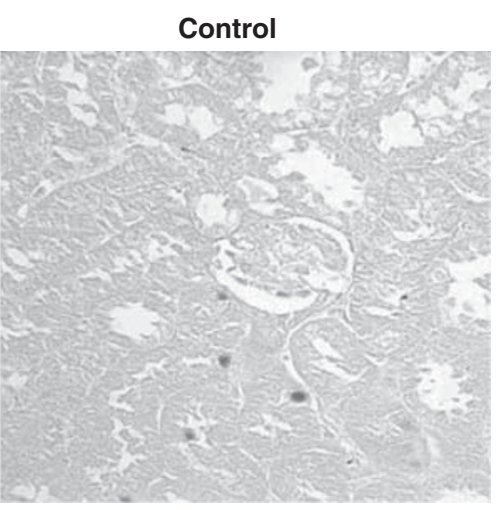

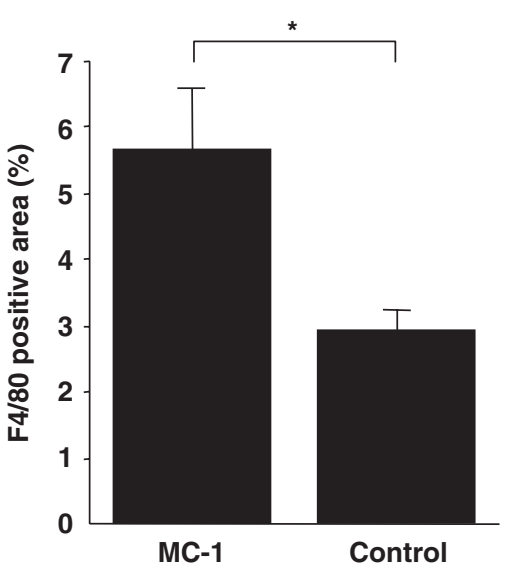

MC-1

Control
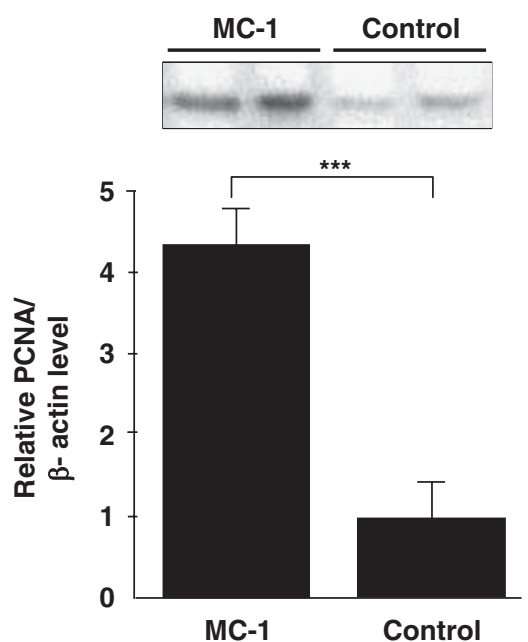

Figure 7 Renal infiltrating and proliferating cells in UUO-R kidneys treated with MC-1 or control for 14 days. (a) (Left) Representative photomicrographs of immunoperoxidase staining for $\alpha$-SMA in MC-1-treated and control mice. (Right) Western blot of $\alpha$-SMA and graph of $\alpha$-SMA level relative to $\beta$-actin level (mean \pm s.e., $n=5,{ }^{*} P<0.01$ ). (b) (Left) Representative photomicrographs of immunoperoxidase staining for F4/80. (Right) Percentage of interstitial area in cortex positive for $F 4 / 80$ (mean \pm s.e., $n=5,{ }^{*} P<0.05$ ). (c) (Left) Representative photomicrographs of immunoperoxidase staining for PCNA. (Right) Western blot of PCNA and graph of PCNA level relative to $\beta$-actin level (mean \pm s.e., $n=5, * * * P<0.001$ ).

ANOVA. Strong induction (20-fold) of MMP-9 by recombinant epimorphin in NRK-49F cells was confirmed at the mRNA level by real-time RT-PCR (Figure 10a).
However, no PA activity band was detected in the culture media of either cell type by plasminogen casein gel zymography (data not shown). 
MC-1

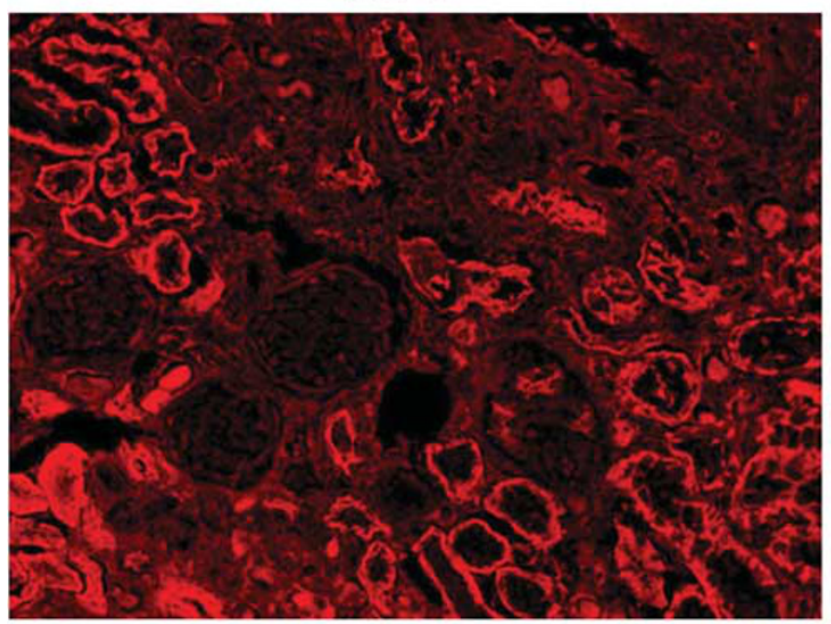

\section{Control}

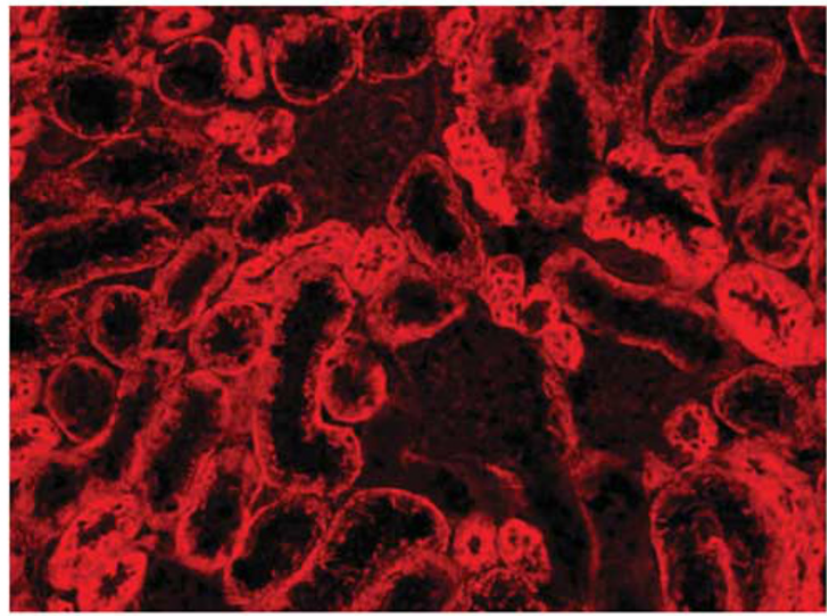

$*$

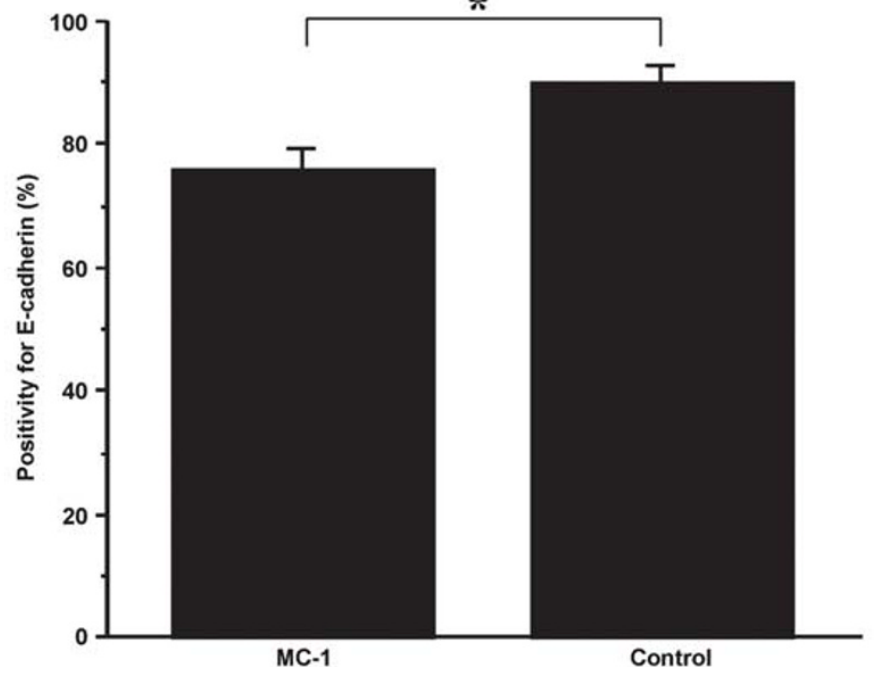

Figure 8 E-cadherin expression on renal tubular epithelial cells in UUO-R kidneys treated with MC-1 or control for 14 days. Representative photomicrographs of immunofluorescence staining for E-cadherin in kidneys from MC-1-treated and control mice (original magnification $\times 400$ ). Bar graphs show percentage of visible tubules that are positive for E-cadherin (mean \pm s.e., $n=5,{ }^{*} P<0.05$ ).

\section{Relation between MMPs and Epimorphin in UUO Kidneys}

Double staining for epimorphin and MMP-9 revealed that the locations of these proteins were quite close and sometimes overlapped in the interstitial area (Figure 10b), suggesting their potential interaction. Although a similar result was yielded by the double staining for MMP-2 and epimorphin, MMP-2 staining was found not only in the interstitial area but also on some tubular epithelial cells (data not shown).

\section{DISCUSSION}

Although epimorphin was first identified as a mesenchymal protein that modulates epithelial morphogenesis in embryonic tissues, ${ }^{4}$ recent studies have shown its importance in the repair process after pathologic organ damage such as lung fibrosis and liver injury. ${ }^{11-13}$ Epimorphin is reported to bind $\alpha \mathrm{V} \beta 5$ and $\alpha \mathrm{V} \beta 1$ integrins on target epithelial cells and to initiate specific signaling processes, ${ }^{32}$ but its receptors and its signaling pathways have not been fully identified and its functions in vivo remain unclear.

In this study, we observed the previously reported localization of epimorphin in the glomerular mesangium, vessel walls and interstitial area in the kidneys of the sham-operated mice and in the contralateral kidneys of the UUO mice. ${ }^{33,34}$ The epimorphin staining was unchanged in the glomerulus but in the tubulointerstitial area it increased prominently with the progression of interstitial fibrosis in the UUO kidneys. It was not found on tubular epithelial cells but was found in the interstitial area, especially around the TBM. Most of interstitial cells positive for epimorphin were $\alpha$-SMA-positive cells (myofibroblasts), and most of the vimentin-positive or FSP-1-positive interstitial cells were negative for epimorphin. We think this is the first study to 

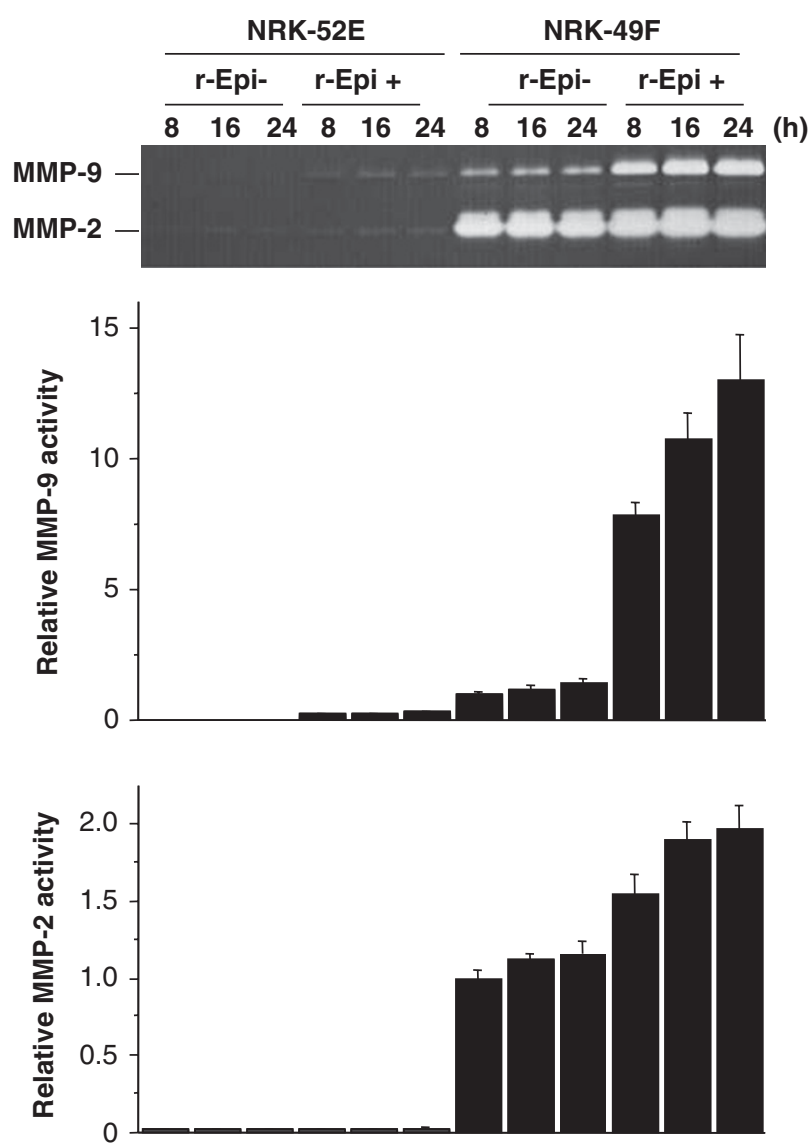

Figure 9 Gelatin gel zymography results showing the effect of recombinant epimorphin on NRK-52E cells (rat renal tubular epithelial cells) and NRK-49F cells (rat renal interstitial fibroblasts) cultivated for 8,16 and $24 \mathrm{~h}$ in serum-free media with $(+)$ or without $(-)$ recombinant epimorphin ( $r$-Epi). Note the prominent induction of MMP-9 in NRK-49F cells. Graphs show means \pm s.e. of triplicate assays.

examine in detail the temporal and spatial changes in the distribution and magnitude of epimorphin expression in diseased kidneys. The enhanced epimorphin expression might indicate that epimorphin has a role in the progression as well as the repair of fibrosis.

In most renal disease models including the UUO mouse, the pathophysiological condition is determined by both the damage mechanisms and the repair mechanisms. However, the relative contributions of these mechanisms to each pathologic condition are not always clear. The UUO-R model is valuable because it let us analyze the endogenous repair mechanisms free from the influence of the damage mechanism. In this study using this model, epimorphin expression was stronger in the early recovery phase than in the injury phase. Furthermore, treatment with antiepimorphin antibody (MC-1) significantly retarded the fibrosis repair in UUO-R kidneys. Thus, epimorphin is prominently upregulated in fibrotic kidneys and is assumed to contribute to the repair process. The finding that epimorphin colocalized well with $\alpha$-SMA-positive cells is somewhat in accordance with this concept because recent reports indicate important roles of $\alpha$-SMA in the repair of renal fibrosis. ${ }^{35,36}$

Syntaxins are the target membrane soluble $\mathrm{N}$-ethylmaleimide-sensitive factor attachment protein receptors (t-SNARE) that mediate intracellular vesicle targeting and fusion. ${ }^{6,37,38}$ Among syntaxin members, epimorphin (syntaxin-2) is the only one reported to exert an effect extracellularly as an epithelial morphogen. To preliminarily analyze the possible roles of other syntaxin members in renal fibrosis, we analyzed the expression of mRNA for syntaxin1a, which shows a very high level of sequence similarity but has no activity as a morphogen, ${ }^{39}$ by real-time RT-PCR. Similar to epimorphin, syntaxin-1a increased over time after UUO (Supplementary Figure 2A). In contrast to epimorhin, syntaxin-1a constantly decreased over time in the kidney of UUO-R mice (Supplementary Figure 2B). These results are consistent with syntaxin-1a not exerting an effect as a morphogen. ${ }^{39}$

Fibrosis is characterized by the accumulation of ECM components, which results from the imbalance between ECM production and degradation. ECM is mainly degraded through two distinct pathways: the MMP degrading pathway and the PA/plasmin proteolytic axis. ${ }^{40}$ In this study, inhibition of epimorphin by antibody prominently decreased the expression of MMP-2 and MMP-9 in UUO-R kidneys. This finding is supported by the effect of recombinant epimorphin on renal interstitial fibroblasts (NRK-49F cells) in vitro. Thus, epimorphin might have induced interstitial fibroblasts to secrete MMP-2 and MMP-9, thereby accelerating ECM degradation and fibrosis repair in UUO-R kidneys. Indeed, as epimorphin and MMPs are located close together (Figure 10b), epimorphin presumably induces interstitial mesenchymal cells to secrete MMP-2 and MMP-9 in vivo. MMPs are essential for normal renal development, ${ }^{41}$ and epimorphin is reported to direct morphogenesis by regulating MMPs. ${ }^{42}$ Thus, MMP-2 and MMP-9 activities upregulated by epimorphin might also induce accelerated regeneration of fibrotic kidneys. The significant delay in E-cadherin expression on tubular epithelial cells in MC-1treated mice (compared with control mice) supports this concept and suggests that endogenous epimorphin induces mesenchymal-epithelial interaction.

PA and plasmin activities were higher in MC-1-treated mice than in control mice. We suspect that the small but significant difference in these activities was not the primary change but rather a change secondary to the difference in tissue repair. MC-1-treated mice had more interstitial monocyte/macrophage infiltration than did control mice, and monocytes and macrophages are known to secrete urokinase-type PA and tissue-type PA. ${ }^{43}$ It is therefore possible that MC-1-treated mice would show more PA/plasmin activity secondary to the greater renal monocyte/macrophage infiltration. In fact, recombinant epimorphin did not induce PA activity on either renal epithelial cells or renal fibroblasts 
b

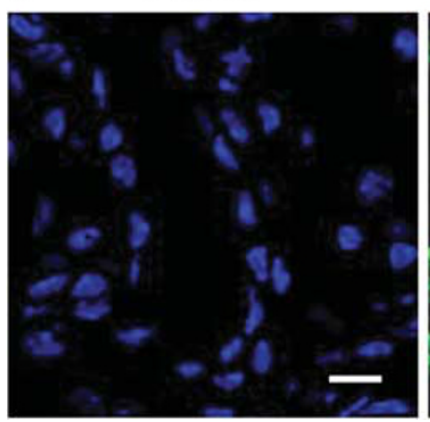

a

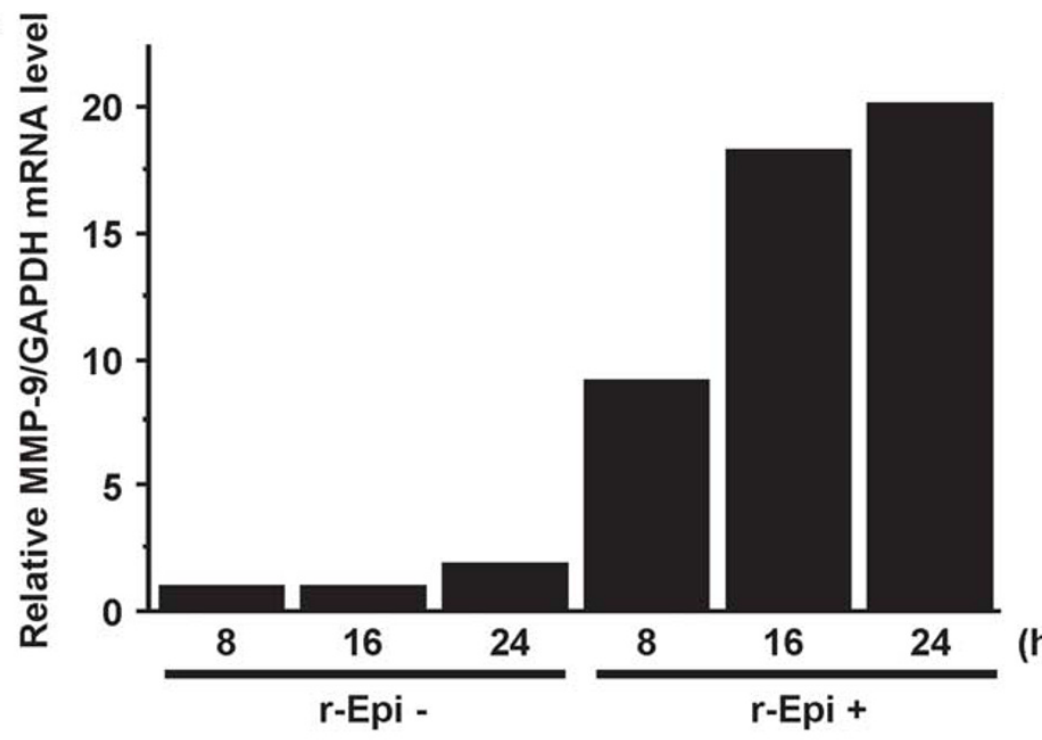

(h)
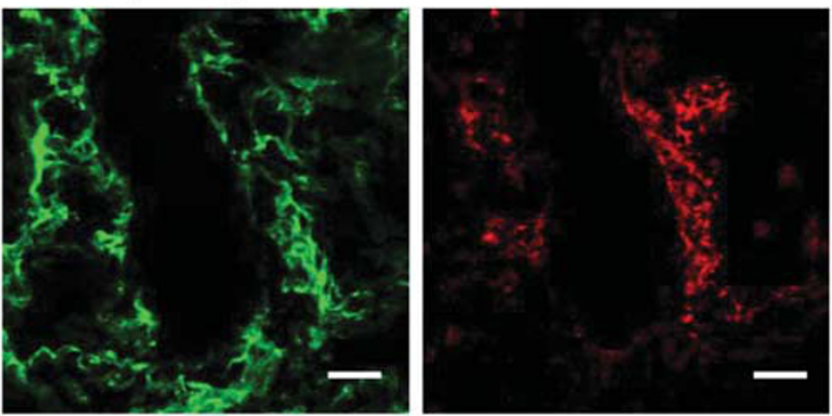

Merged image

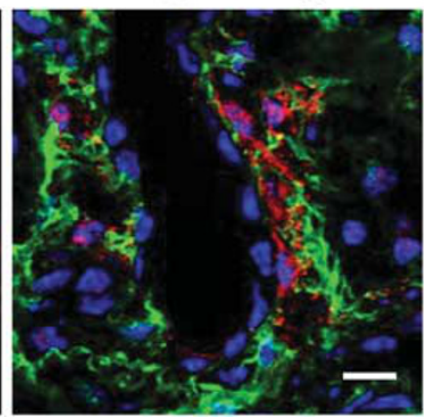

Figure 10 Relation of MMP-9 and epimorphin in vitro and in vivo. (a) Graph of MMP-9 mRNA levels (relative to GAPDH mRNA levels, both assessed by real time RT-PCR) in culture media of NRK-49F incubated for 8,16 or $24 \mathrm{~h}$ with $(+)$ or without $(-)$ recombinant epimorphin ( $\mathrm{r}$-Epi). (b) Confocal microscopic images of multiple immunofluorescence staining for nuclei, epimorphin and MMP-9 in mouse kidney at 10 days after UUO. Epimorphin-positive area was localized adjacent to MMP-9-positive area in the interstitium of the UUO kidney. Scale bar $=10 \mu \mathrm{m}$.

in vitro (data not shown), suggesting that epimorphin has no direct effect on $\mathrm{PA} /$ plasmin activity.

TGF- $\beta$ is a key mediator in kidney fibrosis and epithelialmesenchymal transition. ${ }^{18,19,21}$ In this study TGF- $\beta$ mRNA expression was significantly higher in MC-1-treated mice than in control mice. Collagen I and III mRNA expression levels were also higher in MC-1-treated mice than in control mice, but the difference in collagen III between groups did not reach statistical difference. Thus, anti-epimorphin antibody seemed to retard the repair of fibrosis not only through downregulation in ECM degradation (suppressed expression of MMP-2 and MMP-9) but also through upregulation in ECM production (enhanced expression of TGF- $\beta$ and collagen I).

The results of immunohistochemistry of UUO-R tissues treated with anti-epimorphin antibody indicate that the difference in ECM production might be due to the difference in the number of interstitial infiltrating cells, such as macrophages and myofibroblasts, but the mechanism by which inhibition of epimorphin induces greater macrophage and myofibroblast infiltration was not examined and deserves future study. Nevertheless, our finding that epimorphin inhibition caused persistently increased macrophage and myofibroblast infiltration, cell proliferation and loss of E-cadherin expression on tubular epithelial cells strongly suggests that epimorphin functions basically in the repair of renal fibrosis possibly through mesenchymal-epithelial interaction. Considering what we know of tissue damage in other organs, ${ }^{11-15}$ we assume that when a kidney is injured epimorphin functions as a repair protein and has a role similar to its role as a morphogen when a kidney develops.

In conclusion, epimorphin has a key role in the repair of renal fibrosis, possibly through mesenchymal-epithelial interaction, by enhancing ECM degradation and decreasing its production. Capitalizing on this role might become important in designing therapeutic strategies for treating or preventing renal interstitial fibrosis.

Supplementary Information accompanies the paper on the Laboratory Investigation website (http://www.laboratoryinvestigation.org) 


\section{ACKNOWLEDGEMENTS}

We are grateful to our colleagues Ms Ryoko Ogata and Ms Yasuko Sugimoto for expert secretarial assistance and to Dr Keiichi Ito, Department of Urology, National Defense Medical College, and Dr Yasuhiro Terasaki, Department of Cell Pathology, Postgraduate School of Medicine, Kumamoto University, for valuable advice and discussion. Parts of this study were presented at the annual meetings of the American Society of Nephrology held in November 2007 and 2008.

\section{DISCLOSURE/CONFLICT OF INTEREST}

The authors declare no conflict of interest.

1. Lysaght MJ. Maintenance dialysis population dynamics: current trends and long-term implications. J Am Soc Nephrol 2002;13(Suppl 1):S37-S40.

2. Liu Y. Renal fibrosis: new insights into the pathogenesis and therapeutics. Kidney Int 2006;69:213-217.

3. Eddy AA. Molecular basis of renal fibrosis. Pediatr Nephrol 2000;15:290-301.

4. Hirai Y, Takebe K, Takashina M, et al. Epimorphin: a mesenchymal protein essential for epithelial morphogenesis. Cell 1992;69:471-481.

5. Hirai $Y$, Lochter A, Galosy $S$, et al. Epimorphin functions as a key morphoregulator for mammary epithelial cells. J Cell Biol 1998;140:159-169.

6. Hirai $Y$, Radisky D, Boudreau $R$, et al. Epimorphin mediates mammary luminal morphogenesis through control of C/EBPbeta. J Cell Biol 2001;153:785-794.

7. Miura $K$, Nagai $H$, Ueno $Y$, et al. Epimorphin is involved in differentiation of rat hepatic stem-like cells through cell-cell contact. Biochem Biophys Res Commun 2003;311:415-423.

8. Lehnert L, Lerch MM, Hirai $Y$, et al. Autocrine stimulation of human pancreatic duct-like development by soluble isoforms of epimorphin in vitro. J Cell Biol 2001;152:911-922.

9. Radisky DC, Hirai Y, Bissell MJ. Delivering the message: epimorphin and mammary epithelial morphogenesis. Trends Cell Biol 2003;13:426-434.

10. Fritsch C, Swietlicki EA, Lefebvre O, et al. Epimorphin expression in intestinal myofibroblasts induces epithelial morphogenesis. J Clin Invest 2002;110:1629-1641.

11. Terasaki Y. Epimorphin in bleomycin-induced pulmonary fibrosis in mice. Chest 2001;120:30S-32S.

12. Terasaki $Y$, Fukuda $Y$, Suga $M$, et al. Epimorphin expression in interstitial pneumonia. Respir Res 2005;6:6.

13. Yoshino R, Miura K, Segawa D, et al. Epimorphin expression and stellate cell status in mouse liver injury. Hepatol Res 2006;34:238-249.

14. Segawa D, Miura K, Goto T, et al. Distribution and isoforms of epimorphin in carbon tetrachloride-induced acute liver injury in mice. J Gastroenterol Hepatol 2005;20:1769-1780.

15. Goyal A, Singh R, Swietlicki EA, et al. Characterization of rat epimorphin/syntaxin 2 expression suggests a role in crypt-villus morphogenesis. Am J Physiol 1998;275:G114-G124.

16. Strutz F, Okada H, Lo CW, et al. Identification and characterization of a fibroblast marker: FSP1. J Cell Biol 1995;130:393-405.

17. Okada H, Strutz F, Danoff TM, et al. Possible mechanisms of renal fibrosis. Contrib Nephrol 1996;118:147-154.

18. Fan JM, Ng YY, Hill PA, et al. Transforming growth factor-beta regulates tubular epithelial-myofibroblast transdifferentiation in vitro. Kidney Int 1999;56:1455-1467.

19. Yang J, Liu Y. Dissection of key events in tubular epithelial to myofibroblast transition and its implications in renal interstitial fibrosis. Am J Pathol 2001;159:1465-1475.

20. Yang J, Shultz RW, Mars WM, et al. Disruption of tissue-type plasminogen activator gene in mice reduces renal interstitial fibrosis in obstructive nephropathy. J Clin Invest 2002;110:1525-1538.

21. Iwano M, Neilson EG. Mechanisms of tubulointerstitial fibrosis. Curr Opin Nephrol Hypertens 2004;13:279-284.

22. Zhang G, Kernan KA, Collins SJ, et al. Plasmin(ogen) promotes renal interstitial fibrosis by promoting epithelial-to-mesenchymal transition: role of plasmin-activated signals. J Am Soc Nephrol 2007;18:846-859.
23. Oda T, Jung YO, Kim HS, et al. PAl-1 deficiency attenuates the fibrogenic response to ureteral obstruction. Kidney Int 2001;60:587596.

24. Mizuno S, Matsumoto K, Nakamura T. Hepatocyte growth factor suppresses interstitial fibrosis in a mouse model of obstructive nephropathy. Kidney Int 2001;59:1304-1314.

25. Cochrane AL, Kett MM, Samuel CS, et al. Renal structural and functional repair in a mouse model of reversal of ureteral obstruction. J Am Soc Nephrol 2005;16:3623-3630.

26. Oda T, Yoshizawa N, Takeuchi A, et al. Glomerular proliferating cell kinetics in acute post-streptococcal glomerulonephritis (APSGN). J Pathol 1997;183:359-368.

27. Omasu F, Oda T, Yamada M, et al. Effects of pioglitazone and candesartan on renal fibrosis and the intrarenal plasmin cascade in spontaneously hypercholesterolemic rats. Am J Physiol Renal Physiol 2007;293:F1292-F1298.

28. Moriyama T, Kawada N, Ando A, et al. Up-regulation of HSP47 in the mouse kidneys with unilateral ureteral obstruction. Kidney Int 1998;54:110-119.

29. Nagatoya K, Moriyama T, Kawada N, et al. Y-27632 prevents tubulointerstitial fibrosis in mouse kidneys with unilateral ureteral obstruction. Kidney Int 2002;61:1684-1695.

30. Oda T, Tamura K, Yoshizawa N, et al. Elevated urinary plasmin activity resistant to alpha2-antiplasmin in acute poststreptococcal glomerulonephritis. Nephrol Dial Transplant 2008;23:2254-2259.

31. lizuka $M$, Sasaki $K$, Hirai $Y$, et al. Morphogenic protein epimorphin protects intestinal epithelial cells from oxidative stress by the activation of EGF receptor and MEK/ERK, PI3 kinase/Akt signals. Am J Physiol Gastrointest Liver Physiol 2007;292:G39-G52.

32. Hirai Y, Nelson CM, Yamazaki K, et al. Non-classical export of epimorphin and its adhesion to alphav-integrin in regulation of epithelial morphogenesis. J Cell Sci 2007;120:2032-2043.

33. Horikoshi S, Yoshikawa M, Shibata T, et al. Protein localization and mRNA expression of epimorphin in mouse and human kidneys. Exp Nephrol 2001;9:412-419.

34. Zhang $L$, Ishikawa $O$, Takeuchi $Y$, et al. Immunohistochemical distribution of epimorphin in human and mouse tissues. Histochem J 1998;30:903-908.

35. Sun DF, Fujigaki Y, Fujimoto T, et al. Mycophenolate mofetil inhibits regenerative repair in uranyl acetate-induced acute renal failure by reduced interstitial cellular response. Am J Pathol 2002;161: 217-227.

36. Takeji M, Moriyama $\mathrm{T}$, Oseto $\mathrm{S}$, et al. Smooth muscle alpha-actin deficiency in myofibroblasts leads to enhanced renal tissue fibrosis. J Biol Chem 2006;281:40193-40200.

37. Spring J, Kato M, Bernfield M. Epimorphin is related to a new class of neuronal and yeast vesicle targeting proteins. Trends Biochem Sci 1993;18:124-125.

38. Sieber JJ, Willig KI, Heintzmann R, et al. The SNARE motif is essential for the formation of syntaxin clusters in the plasma membrane. Biophys $J$ 2006;90:2843-2851.

39. Chen CS, Nelson CM, Khauv D, et al. Homology with vesicle fusion mediator syntaxin-1a predicts determinants of epimorphin/syntaxin-2 function in mammary epithelial morphogenesis. J Biol Chem 2009;284:6877-6884.

40. Gong R, Rifai A, Tolbert EM, et al. Hepatocyte growth factor modulates matrix metalloproteinases and plasminogen activator/plasmin proteolytic pathways in progressive renal interstitial fibrosis. J Am Soc Nephrol 2003;14:3047-3060.

41. Lenz O, Elliot SJ, Stetler-Stevenson WG. Matrix metalloproteinases in renal development and disease. J Am Soc Nephrol 2000;11:574-581.

42. Simian M, Hirai Y, Navre M, et al. The interplay of matrix metalloproteinases, morphogens and growth factors is necessary for branching of mammary epithelial cells. Development 2001;128:3117-3131.

43. Oka H, Kugiyama $\mathrm{K}$, Doi $\mathrm{H}$, et al. Lysophosphatidylcholine induces urokinase-type plasminogen activator and its receptor in human macrophages partly through redox-sensitive pathway. Arterioscler Thromb Vasc Biol 2000;20:244-250. 